\title{
DIAGNOSIS OF IMPORTED MALARIA BY PLASMODIUM LACTATE DEHYDROGENASE (pLDH) AND HISTIDINE-RICH PROTEIN 2 (PfHRP-2)-BASED IMMUNOCAPTURE ASSAYS
}

\author{
JAMSHAID IQBAL, PARSOTAM R. HIRA, ALI SHER, AND ABDUL AZIZ AL-ENEZI
}

Department of Microbiology, Faculty of Medicine, Kuwait University, Safat, Kuwait; Malaria Laboratory, Ministry of Health, Safat, Kuwait; and Infectious Diseases Hospital, Ministry of Health, Safat, Kuwait

\begin{abstract}
This study was conducted to evaluate the performance of two rapid non-microscopic assays: Plasmodium lactate dehydrogenase (pLDH) assay (OptiMAL) and Plasmodium falciparum histidine-rich protein 2 (PfHRP-2) assay (ICT Malaria). The assays were used to detect malaria infection in 515 immigrants living in Kuwait. The performance of both assays was compared to that of microscopy of Giemsa-stained thick blood films and to each other. Of the 515 patients tested, 163 were positive for malaria parasites by microscopy of thick blood film. Of these, 87 were infected with Plasmodium vivax parasites, 63 with $P$. falciparum, 1 with Plasmodium malariae, and 12 had mixed infections of $P$. falciparum and $P$. vivax. The PfHRP-2 assay detected $53 P$. falciparum infections and, as expected, failed to detect all but one case of $P$. vivax. Three cases of mixed infections were also not detected by this assay. The pLDH assay detected $56 P$. falciparum cases and $77 P$. vivax infections but failed to detect 4 cases of mixed infections. Compared to microscopy, the performance of both the assays to diagnose $P$. falciparum infection was comparable. The sensitivity for the PfHRP-2 assay was $82 \%$ with a specificity of $99.0 \%$ and for the pLDH assay the sensitivity was $89 \%$ with a specificity of $99.5 \%$. The PfHRP-2 assay detected 4 false positive cases, 2 of which were also detected by the pLDH assay. These patients reported treatment with chloroquine in the last 2-5 weeks. Though the immunocapture diagnostic assays may be helpful in certain situations, microscopy of thick blood film is still the method of choice in diagnosing imported malaria.
\end{abstract}

\section{INTRODUCTION}

Early diagnosis and treatment of parasitemia is vital for the control of malaria, one of the most prevalent parasitic diseases worldwide. Microscopic examination of thick blood film is currently the standard method for malaria diagnosis. This method is relatively simple and has low direct costs, but its reliability and cost-effectiveness are questionable, particularly at low levels of parasitemia, i.e., density and in the interpretation of mixed infection. ${ }^{1,2}$ Recently, three rapid non-microscopical assays for the detection of Plasmodium falciparum infection have been developed. These tests are based on the detection of antigen(s) released from parasitized red blood cells. Two of the assays, ParaSight F (Becton Dickinson, Cockeysville, MD) and the ICT Malaria Pf (ICT Diagnostics, Sydney, Australia), detect Plasmodium falciparum histidine-rich protein-2 (PfHRP-2). ${ }^{3-5}$ Plasmodium falciparum histidine-rich protein is a water-soluble antigen expressed by trophozoites of $P$. falciparum and by immature gametocytes. However, since PfHRP-2 is only produced by $P$. falciparum, these tests cannot detect infections with Plasmodium vivax, Plasmodium ovale, or Plasmodium malariae. The third test, pLDH assay (OptiMAL) (Flow Inc., Portland, OR) is relatively new and detects a different malarial antigen, Plasmodium-specific lactate dehydrogenase (pLDH). ${ }^{6}$ Unlike the PfHRP-2 assays, the pLDH assay can be used to detect infections with any of the Plasmodium species that infect humans. Both ICT and ParaSight F tests have been evaluated for their sensitivity in detecting malaria infection at various epidemiological settings; however, these tests have not been compared and evaluated for their sensitivity against pLDH assay to detect $P$. falciparum infection.

The present study examined the performance of the PfHRP-2 assay (ICT Malaria) and pLDH assay (OptiMAL) for detecting imported malaria infection in patients returning to Kuwait from countries endemic for malaria. Both assays were compared with the microscopy of thick blood film and with each other.

\section{MATERIALS AND METHODS}

From January 1997 to June 1998, blood specimens were collected from 515 individuals who presented with fever at Mubarek Al-Kabeer Teaching Hospital, and at the Malaria Laboratory, Kuwait. The majority of these individuals were immigrants from tropical countries where malaria infection is endemic. These individuals had arrived in Kuwait in the previous $2-6$ months.

Information about recent treatment for malaria was collected from all of the patients, and a urine specimen was taken for the detection of 4-aminoquinolines (Dill-Glazko) and sulphonamides (lignin). The tests were performed either at the Malaria Laboratory, Ministry of Health, or at the Faculty of Medicine, Kuwait University. Both the PfHRP-2 and pLDH assays were performed blind. Informed consent was taken from all the patients included in the study. The study was approved by the ethical committee of the Faculty of Medicine, University of Kuwait.

Microscopy of Giemsa-stained blood films. Thick and thin blood films were stained with $10 \%$ Giemsa stain for 10 minutes and examined by two experienced microscopists who had no knowledge of patient disease status or nationality to avoid any bias in blood-film readings. The microscopist counted a minimum of 200 consecutive fields in the thick blood film before classifying a slide as negative. Parasites in thick blood film were counted against 200-500 white blood cells (WBCs). The parasite density was estimated assuming 8,000 WBC/ $\mu \mathrm{L}$ of blood..$^{2,7}$

Malaria diagnosis with pLDH assay (OptiMAL). The pLDH assay was performed following manufacturer's in- 
TABLE 1

Performance of the PfHRP-2 and pLDH assays in 515 immigrants*

\begin{tabular}{|c|c|c|c|c|c|}
\hline & \multicolumn{2}{|c|}{ No. positive by } & \multirow{2}{*}{\multicolumn{2}{|c|}{$\begin{array}{c}\text { No. } \\
\text { positive } \\
\text { by pLDH } \\
\text { Ratio } \dagger \\
\text { assay }\end{array}$}} & \multirow[b]{2}{*}{ Ratioł } \\
\hline & $\begin{array}{l}\text { Thick } \\
\text { film }\end{array}$ & $\begin{array}{c}\text { PfHRP-2 } \\
\text { assay }\end{array}$ & & & \\
\hline P. falciparum & 63 & 53 & & 56 & \\
\hline P. vivax & 87 & 1 & & 77 & \\
\hline P. malariae & 1 & 0 & & 1 & \\
\hline Mixed infection & 12 & 9 & & 8 & \\
\hline Total positives & 163 & 63 & 0.39 & 142 & 0.87 \\
\hline Total negatives & 352 & 452 & & 373 & \\
\hline \multicolumn{6}{|l|}{ P. falciparum only } \\
\hline Trophozoites§ & 66 & 58 & 0.88 & 61 & 0.92 \\
\hline$>100 / \mu 1$ & 41 & 39 & 0.95 & 40 & 0.98 \\
\hline$<100 / \mu 1$ & 25 & 19 & 0.76 & 21 & 0.84 \\
\hline Gametocytes & 9 & 4 & & 3 & \\
\hline Trophozoites of all species & 154 & 59 & 0.38 & 139 & 0.90 \\
\hline
\end{tabular}

structions. Briefly, 1 drop of whole blood was mixed with 2 drops of lysis buffer A, which disrupts the red blood cells and releases the $\mathrm{pLDH}$, and the specimens were allowed to migrate to the top of the pLDH strip. After 8 minutes, the strip was cleared by adding 2 drops of buffer B. Interpretation of the test results was performed immediately. A negative-control sample taken from an individual who had not been exposed to malaria for three years, was included with each batch tested. In the pLDH assay there are two diagnostic zones of reaction containing different antibodies. A monospecific antibody that recognizes only P. falciparum is present in the bottom reaction zone. A second pan-specific antibody is present immediately above this zone. This monoclonal antibody recognizes the pLDH isoforms of $P$. vivax. A third reaction zone containing a pan-specific monoclonal antibody is present at the top of the test strip and serves as a positive control for the assay.

Malaria diagnosis with the PfHRP-2 assay. The PfHRP2 (ICT Malaria Pf) assay uses 2 monoclonal antibodies specific for PfHRP-2 antigen. One of the antibodies is attached to visible colloidal gold and impregnated into an absorbent strip, while the second antibody is immobilized in a line across the test strip. The test was performed following manufacturer's instructions. Briefly, $10 \mu \mathrm{L}$ of whole blood is added to the test strip, where lysis occurs and any PfHRP-2 antigen present binds to the colloidal gold-labeled antibody. On adding buffer to the strip, the blood and labeled antibody migrate up the test strip, crossing the second antibody line. In a positive sample, PfHRP-2 complexed with the goldlabeled antibody is captured by the antibody on the membrane and a pink line forms. In a negative sample, no pink line appears.

The sensitivity was calculated as the proportion of positive results obtained among samples containing $P$. falciparum trophozoites; the specificity was calculated as the proportion of negative results obtained among samples whose thick blood films were negative or contained $P$. falciparum gametocytes only. Positive and negative predictive values were calculated as the proportion of true-positive results
TABLE 2

Sensitivity and specificity of the PfHRP-2 and pLDH assays for Plasmodium falciparum trophozoites only in 515 immigrants*

\begin{tabular}{|c|c|c|}
\hline & PfHRP-2 assay & pLDH assay \\
\hline True positive $\dagger$ & 54 & 59 \\
\hline True negative $\neq$ & 445 & 447 \\
\hline False positive $\S$ & 4 & 2 \\
\hline False negativedI & 12 & 7 \\
\hline
\end{tabular}

among all positive reactors and as the proportion of truenegative results among all negative reactors, respectively.

\section{RESULTS}

Of the 515 patients examined, 163 were positive for malaria parasites by microscopy of thick blood films: 87 were infected with $P$. vivax parasites, 63 with $P$. falciparum, 1 with $P$. malariae, and 12 had mixed infections of $P$. falciparum and $P$. vivax. Nine cases had $P$. falciparum gametocytes only (Table 1 ).

The PfHRP-2 assay detected PfHRP-2 antigen in $53 P$. falciparum patients and in 1 case with $P$. vivax infection; however, it was unable to detect 3 patients with mixed infections and 5 patients with gametocytes only (Table 1). Considering P. falciparum trophozoites only, the PfHRP-2 assay detected PfHRP-2 antigen in 58 patients, but failed to detect infection in 2 patients with high parasitemia ( 970 and 1,800 parasites $/ \mu \mathrm{L}$ ) and in 6 patients with parasitemia of $<$ $100 / \mu \mathrm{L}$ (Table 1 ). The pLDH assay detected 56 P. falciparum infections but failed to detect mixed infections in 4 patients and in 6 patients with gametocytes only (Table 1). Considering $P$. falciparum trophozoites only, the pLDH assay detected infection in 61 patients; however, it failed to detect infection in 1 patient with high parasitemia $(1,450$ parasites $/ \mu \mathrm{L})$ and in 4 patients with parasitemia $<100 / \mu \mathrm{L}$ (Table 1). The data presented suggest that both PfHRP-2 and pLDH assays are useful in detecting single infections, but failed to detect majority $(>70 \%$ ) of mixed $P$. falciparum and $P$. vivax infections.

Comparing PfHRP-2 and pLDH assays with the microscopy for the detection of $P$. falciparum trophozoites only, both the PfHRP-2 and pLDH assays failed to detect malaria antigen in 12 and 7 microscopy-positive cases, respectively; the majority of these cases had parasitemia $<40 / \mu \mathrm{L}$ (Table 2). There was one PfHRP-2 positive but $\mathrm{pLDH}$ negative case. However, there were three cases which were positive with $\mathrm{pLDH}$ assay but negative with the PfHRP-2 assay; two of these three cases had parasitemia $<40 / \mu \mathrm{L}$. The PfHRP2 assay detected malaria infection in 4 cases that were negative by microscopy; 2 of these 4 cases were also positive with pLDH assay. Three of these 4 cases reported treatment with chloroquine in the precious $3-5$ weeks. The sensitivity and specificity of both the PfHRP-2 and pLDH assays were comparable to that of microscopy which was used as the standard test. The sensitivity of the PfHRP-2 assay was $82 \%$, the specificity $99 \%$, the positive predictive value $93 \%$, and 
TABLE 3

Specificity and sensitivity of the PfHRP-2 and pLDH assays for Plasmodium falciparum trophozoites only in 515 patients*

\begin{tabular}{|c|c|c|c|c|}
\hline \multirow[b]{2}{*}{ Microscopy } & \multicolumn{2}{|c|}{ PfHRP-2 assay } & \multicolumn{2}{|c|}{ pLDH assay } \\
\hline & Negative & Positive & Negative & Positive \\
\hline Positive末 (66) & 12 & 54 & 7 & 59 \\
\hline Negative $\neq$ (449) & 445 & 4 & 447 & 2 \\
\hline Total tested (515) & 457 & 58 & 454 & 61 \\
\hline
\end{tabular}

the negative predictive value was $97 \%$. The sensitivity of the pLDH assay was $89 \%$, specificity $99.5 \%$, positive predictive value $97 \%$, and negative predictive value was $98 \%$ (Tables 2 and 3).

\section{DISCUSSION}

Each year more than 800 cases of imported malaria are detected in Kuwait among the immigrant population who visit their home countries in southeast Asia (India, Bangladesh, Sri Lanka, Pakistan) to spend holidays. ${ }^{8}$ Both $P$. falciparum and $P$. vivax infections are frequently encountered in Kuwait. In this study 87 of the 163 malaria cases had $P$. vivax infection and 12 cases had mixed infections of $P$. falciparum and $P$. vivax. As expected, the PfHRP-2 assay which detects PfHRP-2 antigen failed to detect all but one case of $P$. vivax infection because PfHRP-2 antigen is released by $P$. falciparum-infected red blood cells only. In most malaria-endemic areas, especially in West Africa, malaria species other than $P$. falciparum amount to less than $5 \%$ of infections, but in imported malaria, as in Kuwait, it is important to detect all species of malaria. Sixty-one percent (99 of 163) of all our malaria cases were infected with $P$. vivax. The sensitivity of the PfHRP-2 assay to detect all malaria infections was only $39 \%$. Considering P. falciparum trophozoites, the sensitivity of PfHRP-2 assay was only $82 \%$ with a specificity of $99 \%$, which is comparable to most earlier studies done to detect $P$. falciparum infections. ${ }^{5,9,10}$

The pLDH assay was more suited to our setting because it can detect both $P$. falciparum and $P$. vivax infections and can differentiate between them. This differentiation is clinically relevant for the proper management of the patients because of the need to initiate radical treatment to avoid relapse of infection. The pLDH assay detected 77 of the 87 $P$. vivax infections and 56 of the $63 P$. falciparum infections with an overall sensitivity of $87 \%$. Considering $P$. falciparum trophozoites only, the sensitivity of $\mathrm{pLDH}$ assay was $89 \%$ with a specificity of $99.5 \%$ in our study; this was comparable to an earlier study which reported a sensitivity of $94 \%$ in detecting $P$. falciparum infection. ${ }^{6}$ The performance of both the PfHRP-2 and pLDH assays was comparable to each other in detecting $P$. falciparum infection; however, the PHRP-2 assay failed to detect, as expected, all but one $P$. vivax infection.

The performance of both the PfHRP-2 and pLDH assays was greatly influenced by the level of parasitemia in peripheral blood. The sensitivity of both the assays was $\geq 95 \%$ at parasitemia $>100 / \mu \mathrm{L}$; however, the sensitivity dropped to
$84 \%$ for the PfHRP-2 assay and to $76 \%$ for pLDH assay at parasitemia $<100 / \mu \mathrm{L}$. In contrast to endemic countries, in imported malaria low parasitemias are frequently responsible for clinical disease. In this study $38 \%$ of the patients with $P$. falciparum infection had parasitemia $<100 / \mu \mathrm{L}$. Both the PfHRP-2 and pLDH assays missed 2 and 1 falciparum malaria cases, respectively with high parasitemia $>900 / \mu \mathrm{L}$. False-negative dipstick test results at higher parasitemias have been noted by others, but the underlying reason is not known. ${ }^{6} 10-14$ We also could not explain the failure to detect some cases with gametocytes only.

Only a small proportion of individuals with negative blood film results were diagnosed as positive by the PfHRP2 assay ( 4 cases) and pLDH assay ( 2 cases). Examination of fever history revealed that 3 had a history of recent treatment with chloroquine. Thus a false positive reaction may occur in individuals who have been recently treated for malaria as reported earlier. ${ }^{3,5,15,16}$ The preliminary data show that PfHRP-2 antigen, which is detected by the PfHRP-2 assay, may persist for up to 7-10 days after asexual parasite clearance,, 515 whereas circulating pLDH activity, which is detected by pLDH assay, drops profoundly immediately after the parasites are cleared from the peripheral blood. ${ }^{17}$ Thus, the pLDH assay may provide the potential to monitor the effectiveness of antimalarial therapy and thus assist in the detection of drug-resistant infections. We are presently investigating this particular role of $\mathrm{pLDH}$ assay in the patient management.

In conclusion, the performance of both the PfHRP-2 and pLDH assays is comparable to that of microscopy to detect $P$. falciparum infection at parasitemia $>100 / \mu \mathrm{L}$; however, the pLDH assay offers an advantage over PfHRP-2 assay as samples infected with $P$. vivax are easily distinguished from those infected with $P$. falciparum and also for the detection of mixed infections of $P$. falciparum and $P$. vivax. In addition, the pLDH assay holds promise for clinical monitoring during the course of malaria infection and treatment. However, thick blood-film examination is still the standard method for diagnosing malaria because it detects all Plasmodium species and offers the clear distinction between parasite growth stages which is essential for making therapeutic decisions.

Acknowledgments: We thank the Ministry of Health, Kuwait, for allowing access to malaria patients.

Financial support: The financial support provided by Kuwait University (MI 109, MI 113) is gratefully acknowledged.

Authors' addresses: Jamshaid Iqbal and Parsotam R. Hira, Department of Microbiology, Faculty of Medicine, Kuwait University, Safat, Kuwait. Ali Sher, Malaria Laboratory, Ministry of Health, Safat, Kuwait. Abdul Aziz Al-Enezi, Infectious Diseases Hospital, Ministry of Health, Safat, Kuwait.

Reprint requests: Dr. Jamshaid Iqbal, Department of Microbiology, Faculty of Medicine, Kuwait University, PO Box. 24923, Safat 13110, Kuwait. Email: iqbal@hsc.kuniv.edu.kw

\section{REFERENCES}

1. Molyneux M, Fox R, 1993. Diagnosis and treatment of malaria in Britain. BMJ 306: 1175-1180.

2. World Health Organization (WHO), 1996. Management of uncomplicated malaria and the use of antimalarial drugs for the 
protection of travelers. Report of an informal consultation. Geneva 18-21 September 1995. WHO/MAL/96; 1075: 98.

3. Beadle C, Long GW, Weiss WR, McElroy PD, Maret SM, Oloo AJ, Hoffman SL, 1994. Diagnosis of malaria by detection of Plasmodium falciparum HRP-2 antigen with a rapid dipstick antigen-capture assay. Lancet 343: 564-568.

4. Garcia M, Kirimoama S, Marlborough D, Leafasia J, Rieckmann KH, 1996. Immunochromatographic test for malaria diagnosis. Lancet 347: 1549.

5. Singh N, Valecha N, Sharma VP, 1997. Malaria diagnosis by field workers using an immunochromatographic test. Trans $R$ Soc Trop Med Hyg 91: 396-397.

6. Palmer CJ, Lindo JF, Klaskala WI, Quesada JA, Kaminsky R, Baum MK, Ager AL, 1998. Evaluation of the OptiMAL test for rapid diagnosis of Plasmodium vivax and Plasmodium falciparum malaria. J Clin Microbiol 36: 203-206.

7. Warhurst DC, Williams JE, 1996. Laboratory diagnosis of malaria: ACP Broadsheet No. 148. J Clin Pathol 49: 533-538.

8. Iqbal J, Sher A, Hira PR, 1998. Malaria in non-endemic Kuwait: detection of very low level of Plasmodium falciparum infections using polymerase chain reaction. Med Principles Practice 7: 277-282.

9. Durrheim DN, la Grange JJP, Govere J, Mngomezulu NM, 1998. Accuracy of a rapid immunochromatographic card test for Plasmodium falciparum in a malaria control programme in South Africa. Trans $R$ Soc Trop Med Hyg 92: 32-33.

10. Van den Ende J, Vervoort T, Van Gompel A, Lynen L, 1998. Evaluation of two tests based on the detection of histidine rich protein 2 for the diagnosis of imported Plasmodium falciparum malaria. Trans $R$ Soc Trop Med Hyg 92: 285-288.
11. Anonymous, 1996. A rapid dipstick antigen capture assay for the diagnosis of falciparum malaria. WHO informal consultation on recent advances in diagnostic techniques and vaccines for malaria. Bull WHO 74: 47-54.

12. Humar A, Harrington MA, Pillai D, Kain KC, 1997. ParaSight ${ }^{\mathrm{TM}}-\mathrm{F}$ test compared with the polymerase chain reaction and microscopy for the diagnosis of $P$. falciparum malaria in travelers. Am J Trop Med Hyg 56: 44-48.

13. Karbwang J, Tasanor O, Kanda T, Wattanagoon Y, Ibrahim M, Na-Bangchang K, Thanavibul A, Rooney W, 1996. ParaSight $F$ test for the detection of treatment failure in multidrug resistant Plasmodium falciparum malaria. Trans $R$ Soc Trop Med Hyg 90: 513-515.

14. Cooke AH, Chiodini PL, Doherty T, Moody AH, Ries J, Pinder, M, 1999. Comparison of a parasite lactate dehydrogenasebased immunochromatographic antigen detection assay (OptiMAL) with microscopy for the detection of malaria parasites in human blood samples. Am J Trop Med Hyg 60: 173176.

15. Shiff CJ, Premji Z, Minjas JN, 1993. The rapid manual ParaSight F test: a new diagnostic tool for Plasmodium falciparum infection. Trans $R$ Soc Trop Med Hyg 87: 646-648.

16. Shiff CJ, Minjas JN, Premji Z, 1994. The ParaSight F test: a simple rapid manual dipstick test to detect Plasmodium falciparum infection. Parasitol Today, 10: 494-495.

17. Piper R, Lebras J, Wentworth L, Hunt-Cooke A, Houze S, Chiodini P, Makler M, 1999. Immunocapture diagnostic assays for malaria using Plasmodium lactate dehydrogenase (pLDH). Am J Trop Med Hyg 60: 109-118. 